\title{
DIVERTÍ́CULOS ESOFÁGICOS EN UNA YEGUA CRIOLLA COLOMBIANA: REPORTE DE CASO
}

\section{ESOPHAGEAL DIVERTICULA IN COLOMBIAN CREOLE MARE: CASE REPORT}

\begin{abstract}
Omar F. Arenas ${ }^{1}$, Gustavo A. Villa ${ }^{2}$, José R. Martínez A. ${ }^{3}$
${ }^{1}$ EST MV. Universidad de Antioquia, Medellín-Colombia, e-mail: omar-are@hotmail.com; ${ }^{2}$ MV. Universidad de Antioquia, práctica privada, Medellín-Colombia, e-mail: tavovilladiaz@hotmail.com; ${ }^{3}$ MVZ, MS, PhD. LIMCE Línea de Investigación en Medicina y Cirugía Equina, Grupo de Investigación Centauro. Escuela de Medicina Veterinaria, Facultad de Ciencias Agrarias. Universidad de Antioquia, calle 70 No. 52-21, Medellín, Antioquia-Colombia, e-mail: jrramonmvz@yahoo.com. Autor de correspondencia: jose.martinez@udea.edu.co
\end{abstract}

Rev. U.D.C.A Act. \& Div. Cient. 18(2): 569-573, Julio-Diciembre, 2015

\section{INTRODUCCIÓN}

Las patologías esofágicas son de carácter multifactorial y se manifiestan después de la compactación de material alimenticio, iniciando con el desarrollo de disfagia, progresando a obstrucción y, finalmente, ruptura de esófago (Jones \& Blikslager, 2005). Casos de obstrucción intraluminal son los más comunes en los equinos y, dentro de éstos, se encuentran, con mayor frecuencia, los divertículos.

Los divertículos esofágicos son hallados en los equinos y pueden ser de origen congénitos y adquiridos. Los adquiridos son de mayor incidencia y son clasificados, como divertículos de tracción y de presión (Yamout et al. 2012). De acuerdo con Stick (2012), el tratamiento consiste en la reducción o en la extirpación, a través de la diverticulectomía, dependiendo de la localización y de la accesibilidad al segmento esofágico comprometido.

El objetivo de este trabajo es reportar el caso de la presencia de divertículos esofágicos en una yegua criolla colombiana de paso fino, que fue atendida en una clínica veterinaria del municipio de Envigado, Antioquia-Colombia.

\section{MATERIALES Y MÉTODOS}

Los datos anamnésicos reportaron historia reciente de Síndrome de abdomen agudo (SAA), por impactación gástrica, tratada con hidratación parenteral, con solución cristaloide isotónica de ringer lactato, a $100 \mathrm{~mL} / \mathrm{kg} /$ día, hidratación enteral, con sonda nasogástrica, cada cuatro horas, por 24 horas, en la cual, se administró de cuatro a seis litros de líquidos, compuesto por dextrosa y electrolitos, a base de cloruro de sodio, citrato de sodio y cloruro de potasio, así como la administración de ranitidina $6,6 \mathrm{mg} / \mathrm{kg} / \mathrm{qid} / \mathrm{iv}$, por tres días. La paciente evolucionó, positivamente, al protocolo instaurado.

El caso corresponde a un equino hembra, criollo colombiano de paso fino, con una condición corporal $7 / 9$, cinco años de edad y un peso de $346 \mathrm{~kg}$. Durante el examen clínico, se reportaron los siguientes signos vitales: frecuencia cardiaca: 38lpm; frecuencia respiratoria: $12 \mathrm{rpm}$; temperatura rectal: $38,7^{\circ} \mathrm{C}$; atenta al medio y con reflejos nerviosos normales y normo-motilidad gastrointestinal; sin embargo, después de 24 horas de estabilidad clínica, posterior al SAA, presentó secreción nasal bilateral mucoide, de forma continua, con contenido de pasto y tendencia a aumentar, al intentar ingerir alimento. De igual forma, evidenció sialorrea, disfagia, odinofagia, estertores traqueales, posición del cuello extendida de forma antero ventral y, a la palpación del área yugular, se encontró enfisema subcutáneo.

Inicialmente, se realizó endoscopia exploratoria -previa sedación con xilacina al $10 \%$, a dosis de $1,1 \mathrm{mg} / \mathrm{kg} / \mathrm{iv}-;$ durante este primer examen, se presentó imposibilidad de entrar al esófago, posiblemente, relacionado con la resistencia de los tejidos inflamados del área; por tal motivo, se instauró tratamiento antiinflamatorio, compuesto de dexametasona a $0,1 \mathrm{mg} / \mathrm{kg} / \mathrm{sid} / \mathrm{iv}$ y flunixin meglumine $1,1 \mathrm{mg} / \mathrm{kg} / \mathrm{bid} / \mathrm{iv}$, ambas durante dos días.

Después de la terapéutica antiinflamatoria, se practicó una segunda endoscopia, bajo sedación con xilacina al 10\%, a dosis de $1,1 \mathrm{mg} / \mathrm{kg} / \mathrm{iv}$ y premedicación con $10 \mathrm{Ul} / \mathrm{iv}$ de oxitocina, 30 minutos antes del procedimiento. La resistencia para 
ingresar al esófago persistió; sin embargo, después de varios intentos, se logró avanzar con el endoscopio, tan solo $3 \mathrm{~cm}$, aproximadamente.

Ante este impedimento, se decidió, inicialmente, retirar el material compactado, con pinzas de uso endoscópico, pero sin éxito, por el poco contenido que se obtenía. Como segunda tentativa -para desobstruir la entrada esofágica-, se introdujo una sonda nasogástrica por el ollar libre, para hidratar y ablandar, con la instilación de líquidos y de dioctilsulfosuccinato sódico (DSS), en el sitio de la obstrucción, para posteriormente realizar tracción mecánica, en sentido aboral. En definitiva, se logró atravesar el sitio obstruido y llegar al estómago, con la sonda, mas no con el endoscopio.

Se efectuó un estudio radiográfico simple del área cervical proximal y, otro, contrastado con sulfato de bario, en el esfínter superior del esófago, en proyecciones latero-lateral. Durante estos procedimientos, se le realizó hidratación parenteral, a razón de $100 \mathrm{~mL} / \mathrm{kg}$ día, con lactato de ringer, sucralfato $15 \mathrm{mg} / \mathrm{kg} /$ qid/vo y se instauró terapia antibiótica conservadora, posterior al procedimiento con enrofloxacina $5 \mathrm{mg} / \mathrm{kg} /$ qid y penicilina G sódica $22.000 U \mathrm{Ul} / \mathrm{kg} /$ qid/iv. Durante el día quinto de evolución clínica, se tomaron muestras de sangre, para hemoleucograma y determinación de BUN, creatinina y sodio.

Ante la persistencia de los signos, se decidió realizar una tercera endoscopia, tres días después de la segunda, en este caso, con protocolo de anestesia general endovenosa fija, compuesta de xilacina al $10 \%$, a dosis de $0,8 \mathrm{mg} / \mathrm{kg} / \mathrm{iv}$, ketamina al $10 \%$, a dosis de $2,2 \mathrm{mg} / \mathrm{kg} / \mathrm{iv}$ y guayacolato de glicerilo al $10 \%$, a dosis de $100 \mathrm{mg} / \mathrm{kg} / \mathrm{iv}$, bajo un esquema de infusión, para mantenimiento de $1 \mathrm{ml} / \mathrm{kg} /$ hora de la solución.

\section{RESULTADOS Y DISCUSIÓN}

La primera endoscopia reportó inflamación del área laríngea, con exacerbación en los procesos corniculados de los cartílagos aritenóides, tal como se muestra en la figura 1A; la exploración endoscópica de la tráquea reportó presencia de moco, que se extendía hasta la carina. El segundo estudio endoscópico evidenció lumen traqueal libre de moco, zona laríngea y cartílagos aritenóides notablemente desinflamados, como se muestra en la figura 1B; igualmente, con el limitado avance del endoscopio, se observaron puntos necróticos y acúmulo de material alimenticio en descomposición. Los hallazgos de la tercera endoscopia fueron similares a los encontrados durante la segunda.

La evaluación radiológica mostró, como hallazgo relevante, cambios de radiolucidez compatible, con acúmulo de gas, en los tejidos circunvecinos ventrales al esófago del área y sin alteraciones en el lumen esofágico; los exámenes de laboratorio, no indicaron alteraciones significativas. Ante la ausencia de mejoría, de pronóstico reservado y de razones económicas del propietario, se tomó la decisión de eutanasia, bajo anestesia general, aplicando $20 \mathrm{~mL}$ de lidocaína $2 \%$ intratecal, según protocolo, propuesto por Amaral et al. (2011).

En la necropsia, se evidenciaron dos divertículos, que iniciaban en el área dorsal de la entrada del esófago y de manera transversal y en forma cónica, recorrían el perímetro hasta el área ventral, sin comunicación entre sí; cada saculación, con un tamaño aproximado de 3 y $4 \mathrm{~cm}$ de profundidad. La cavidad de los divertículos contenía partículas de alimento impactado, que ejercían presión hacia la luz del esófago.

Muestras de tejido de las saculaciones de la pared esofágica fueron tomadas y procesadas, para estudio histopatológico. Necrosis, inflamación crónica activa con reparación, inflamación flegmonosa y gangrenosa severa -con extensión a capas profundas, como lámina propia, submucosa, túnica muscular y adventicia del esófago-, necrosis de Zenker, necrosis grasa y microabscesos - de diferente tamaño-, se reportaron microscópicamente.

Los divertículos esofágicos adquiridos son los más comúnmente encontrados en los equinos, mientras los de naturaleza congénita son de escasa presentación, de acuerdo a lo reportado por Stick (2012); sin embargo, este reporte describe una alteración de tipo congénita, por la configuración bilateral de las saculaciones en el segmento inicial del esófago y la cronicidad de las lesiones halladas macroscópica e histológicamente.

La manifestación clínica del cuadro esofágico, en este equino adulto, a pesar de ser congénito, indicó una lenta y progresiva compactación de material alimentario dentro las cavidades de los divertículos, alcanzando un volumen considerable para disminuir el espacio luminal y afectar el paso de ingesta y alterar la fase esofágica de la deglución. Esta situación, también influyó en la resistencia evidenciada al paso del endoscopio, para realizar la esofagoscopia completa, en esta paciente.

La oclusión luminal y la pérdida de la función esofágica confirmó la presentación de los signos percibidos en la evaluación clínica inicial, que son propios de cuadros obstructivos de este segmento del sistema digestivo del equino. Como consecuencia de la disfagia, la odinofagia y la pérdida constante de saliva, se pueden generar desequilibrios hidroelectrolíticos y ácido-base (Southwood, 2014), cuya severidad depende del tiempo de evolución e intervención médica. Hiponatremia, hipocloremia y pérdida de bicarbonato por salivación conducen a acidosis metabólica transitoria (Feige et al. 2000); sin embargo, en esta paciente, solamente la 


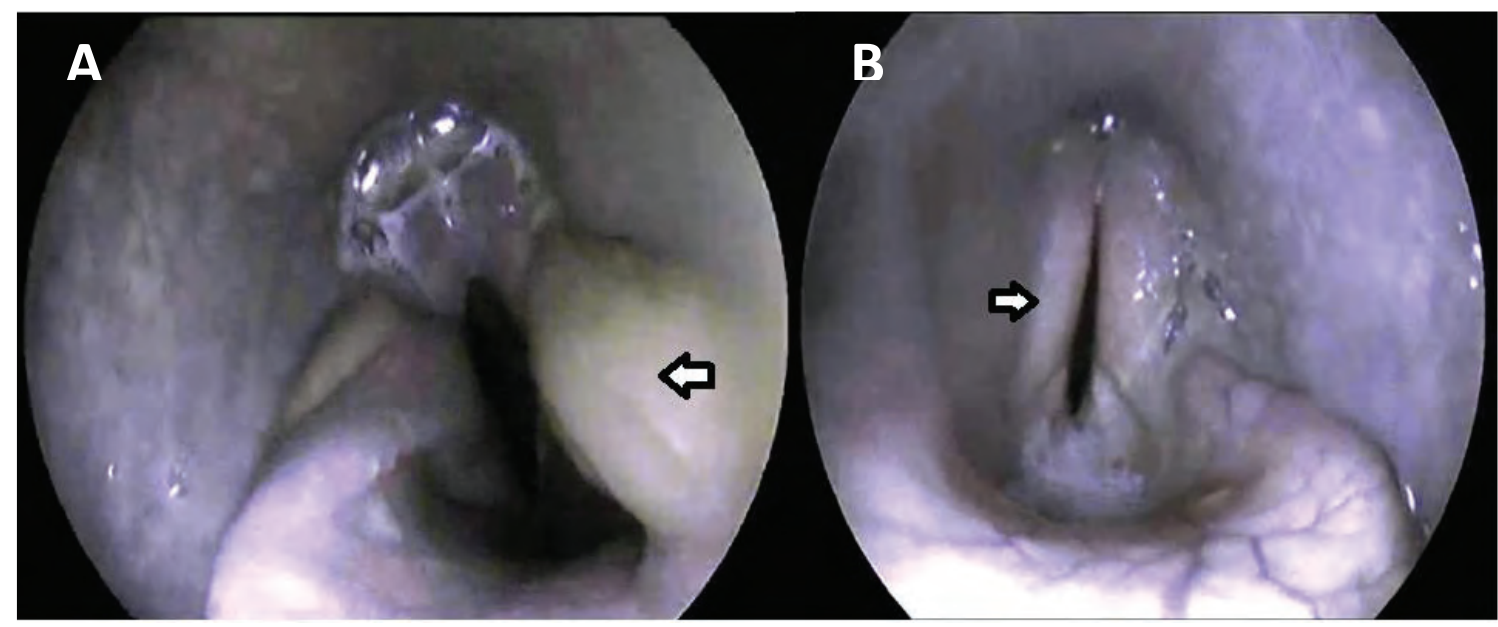

Figura 1. Endoscopia del área laríngea. Inflamación de los cartílagos aritenóides, como indica la flecha blanca y presencia de moco (A). Endoscopia de la misma área después de la terapia antiinflamatoria, con notable respuesta, como lo muestra la flecha blanca (B).

concentración de sodio sanguíneo se reportó en el rango inferior normal. Posiblemente por el monitoreo y la atención inmediata, no se evidenciaron clínicamente ni por laboratorio, estos desequilibrios.

El tratamiento de soporte administrado al inicio y durante el diagnóstico, posiblemente, minimizó las manifestaciones clínicas, como fue evidenciado con los hallazgos normales, en la mayoría de los parámetros evaluados. En este sentido y de acuerdo con Chiavaccini \& Hassel (2010), algunas variables clínicas, junto a resultados endoscópicos, son herramientas importantes para determinar severidad de lesiones esofágicas y compromiso pulmonar; sin embargo, la paciente presentó leve hipertermia, probablemente, relacionado con el estrés o el dolor, ya que la frecuencia respiratoria y el leucograma se registraron normales, lo que descartó un cuadro infeccioso, a pesar de la presencia de secreción en el lumen traqueal, observado a través de la endoscopia.

A causa de la necrosis por presión desarrollada en los divertículos, se produjo perforación o rotura esofágica, causando una extensa celulitis y necrosis de los tejidos adyacentes, con posterior formación de enfisema, como se evidenció en la palpación y en el estudio radiológico (Jones \& Blikslager, 2005). Las placas radiográficas simples mostraron áreas de radiolucidez, debido al gas entre los tejidos periesofágicos; sin embargo, proyecciones radiográficas con contraste fueron necesarias, para descartar la existencia de algún patrón obstructivo en esta paciente, de acuerdo con lo reportado por Greet (1982).
La tentativa inicial de realizar extracción del material impactado dentro los divertículos con las pinzas endoscópicas falló, a causa de varios factores, como la complejidad de la zona afectada, por ser una área de acceso mínimo, así como también, el tamaño de la pinza y la consistencia del material compactado. Igualmente, el lavado y la disgregación propulsiva del esófago permitieron -con dificultad- el paso de la sonda en dirección al estómago, por la mayor consistencia comparada con la del extremo del endoscopio y fue poco exitoso, debido a que la compactación del divertículo comprimió la pared y el lumen esofágico, impidiendo el tránsito aboral por la obstrucción.

El manejo médico instaurado, inicialmente, a base de antibióticos y de analgésicos, son los recomendados por la literatura para casos de obstrucción luminal simple (Feige et al. 2000; Jones \& Blikslager, 2005; Smith, 2010). Posiblemente, la indicación de estos medicamentos contribuyó en evitar el desarrollo de complicaciones infecciosas de tipo respiratorias, por ejemplo, por ser una de las más comunes, como lo describieron Chiavaccini \& Hassel (2010).

La implementación de oxitocina en procura de producir relajación esofágica para facilitar la desobstrucción, se indicó por ser efectiva, en casos donde se compromete la parte inicial o craneal del esófago de pacientes no complicados, de acuerdo a lo reportado por Meyer et al. (2000); a pesar del variable y controversial éxito, se utilizó, en este caso, por la condición clínica presentada; seguramente, el acceso a la entrada del esófago en la segunda endoscopia pudo haber sido por el efecto esperado de este medicamento. El uso de 
sucralfato obedeció a los efectos protectores y cicatrizantes de la mucosa esofágica descritos (Jones \& Blikslager, 2005); sin embargo, por el desenlace del caso, no fue posible evaluar su utilidad.

Los casos de obstrucción esofágica luminal por compactación simple, se caracteriza, por lo general, de tener un buen pronóstico; no obstante, es malo a reservado en casos de obstrucciones, por anormalidades funcionales o morfológicas, como el reportado en este trabajo. En un estudio realizado en 11 caballos con alteraciones esofágicas, sólo dos sobrevivieron a largo plazo, mientras que en otro trabajo, cinco de nueve caballos, fueron sacrificados después de complicaciones esofágicas por sondaje nasogástrico (Hardy et al. 1992). Complicaciones posterapéutica, como la estenosis con potencial de reincidir en obstrucción, se ha estimado en el 37\% de los casos, con 24 horas de duración (Craiq et al. 1989). En este caso, se destacó su cronicidad por ser congénito, con un curso de manifestación clínica por siete días de duración y la baja respuesta al manejo inicial; podría indicar complicaciones mayores en su evolución.

El manejo quirúrgico de alteraciones esofágicas, en la mayoría de los casos, termina con complicaciones, como estenosis y dehiscencia de sutura, por la deficiente cicatrización derivada de características histológicas y anatómicas, particulares de este órgano (Orsini et al. 1991; Godoy, 2009), además de factores extrínsecos, como movilidad y efecto químico de la saliva, que comprometen el proceso de resolución (Stick, 2012). En el caso particular de la paciente de este reporte, el pronóstico se consideró reservado, por lo anteriormente mencionado y por la naturaleza y la compleja ubicación de la anormalidad diagnosticada.

Finalmente, en la decisión de eutanasia influyeron varios factores, relacionados con el tipo y la ubicación de la anormalidad esofágica; el relativo éxito quirúrgico y las razones presupuestales del propietario, para atender el manejo postoperatorio, dado que se requiere modificaciones de dieta y de prevención de posibles complicaciones, tanto en el sitio de lesión como en otros órganos circunvecinos o sistémicos; además de la posibilidad de consecuencias permanentes en la deglución, que puede terminar con el desarrollo de neumonía por aspiración, en un alto porcentaje de los casos (Jones \& Blikslager, 2005). De igual forma, pueden desarrollar cuadros de hemiplejia laríngea, ruptura de la arteria carótida, infecciones postoperatorias, pleuritis, laminitis y síndrome de Horner, según lo reportado por Craiq et al. (1989) y Stick (2012).

En conclusión, los divertículos esofágicos congénitos en los equinos son raros y pueden cursar con una evolución crónica, que se puede agudizar con manifestación clínica y desencadenar graves trastornos. En conocimiento de los au- tores, este reporte de caso es el primero que se estudia y se describe en un ejemplar equino criollo colombiano.

Agradecimientos: Estrategia de sostenibilidad CODI 20132014, Universidad de Antioquia. Conflicto de intereses: El manuscrito fue preparado y revisado con la participación de todos los autores, quienes declaramos que no existe conflicto de intereses que ponga en riesgo la validez del manuscrito presentado.

\section{BIBLIOGRAFÍA}

1. AMARAL, L.C.; RABASSA, V.; MARCHIORI, M.; MEIRELLES, M.G.; AMADO, M.; NOGUEIRA, C.E.W. 2011. Utilizacao de lidocaina $2 \%$ por via intratecal associado a anestesia previa com tiopental sódico como método de eutanasia em equinos. ARS Veterinaria. (Brasil). 27:011-016.

2. CHIAVACCINI, L.; HASSEL, D.M. 2010. Clinical features and prognostic variables in 109 horses with esophageal obstruction (1992-2009). J. Vet. Intern. Med. (USA). 24:1147-1152.

3. CRAIQ, D.R.; SHIVY, D.R.; PANKOWSKI, R.L.; ERB, H.N. 1989. Esophageal disorders in 61 horses. Results of nonsurgical management. Vet. Surg. (USA). 18(6):432-438.

4. FEIGE, K.; SCHWARZWALD, C.; FÜRST, A.; KASERHOTZ, B. 2000. Esophageal obstruction in horses: a retrospective study of 34 cases. Can. Vet. J. (Canada). 41:207-210.

5. GODOY, P.A. 2009. Obstrucción esofágica en equinos (choke). Rev. Tecno.Vet. (Chile). 15(1):17-21.

6. GREET, T.R. 1982. Observations on the potential role of esophageal radiography in the horse. Equine Vet. J. (England). 14:73-79.

7. HARDY, J.; STEWART, R.H.; BEARD, W.L.; YVORCHUKST, J. 1992. Complications of nasogastric intubation in horse: nine cases. J. Am. Vet. Med. Assoc. (USA). 201(3):483-486.

8. JONES, S.L.; BLIKSLAGER, A.T. 2005. Esophageal diseases. En: Reed, S.M.; Bayly, W.M.; Sellon, D.C. (eds) Equine Internal Medicine. Ed. Saunders (USA). p.855-863.

9. MEYER, G.A.; RASHMIR-RAVEN, A.; HELMS, R.J.; BRASHIER, M. 2000. The effect of oxytocin on contractility of the equine oesophagus: a potential treatment for 
oesophageal obstruction. Equine Vet. J. (England). 32:151-155.

10. ORSINI, J.A.; DIKES, N.; RUGGLES, A.; CHARLTON, C.; PERRY, R. 1991. Use of gastrotomy to relieve esophageal obstruction in a horse. JAVMA (USA). 198(2):295-296.

11. SMITH, B.P. 2010. Medicina interna de grandes animales. Elsiever Mosby (ESPAÑA). p.690-694.

12. STICK, A.J. 2012. Esophagus. En: Auer, A.J.; Stick, A.J. (eds). Equine Surgery. Ed. Saunders Elsevier (USA). p.367-388.
13. SOUTHWOOD, L.L. 2014. Digestive system and peritoneal disease. En: Southwood, L.L.; Wilkins, A.P. (eds). Equine emergency and critical care medicine. Ed. Manson Publishing (USA). p.27-154.

14. YAMOUT, S.Z.; MAGDESIAN, K.G.; TOKARZ, D.A.; LE JEUNE, S.S. 2012. Intrathoracic pulsión diverticulum in a horse. Can. Vet. J. (Canada). 53:408-411.

Recibido: Agosto 31 de 2015

Aceptado: Octubre 14 de 2015

Cómo citar:

Arenas, O.F.; Villa, G.A.; Martínez A., J.R. 2015. Divertículos esofágicos en una yegua criolla colombiana: reporte de caso. Rev. U.D.C.A Act. \& Div. Cient. 18(23): 569-573. 